\title{
The histology of prostate tissue following prostatic artery embolization for the treatment of benign prostatic hyperplasia
}

George Camara-Lopes, Romulo Mattedi, Alberto A. Antunes, Francisco C. Carnevale, Giovanni G. Cerri, Miguel Srougi, Venancio A. Alves, Katia R. M. Leite

Department of Pathology (GCL, RM, VAA), Department of Radiology (AAA, MS, KRML) and Department of Urology FCC, GGC), Laboratory of Medical Research, LIM55, University of Sao Paulo Medical School, SP, Brazil

\section{ABSTRACT}

Objective: Prostatic artery embolization (PAE) for the treatment of patients with symptomatic benign prostatic hyperplasia (BPH) is believed to be a safe procedure with a low risk of adverse side effects. Artery embolization is a viable treatment option in patients who are refractory to the classic noninvasive treatments. Knowledge of the histological characteristics of prostate tissue following the procedure is still limited. In this study, we describe the microscopic aspects of the prostate following PAE for BPH.

Materials and Methods: Two patients underwent transurethral resections of the prostate (TURP) after PAE. Embolizations were performed under local anesthesia with an initial pelvic angiography to evaluate the iliac vessels and the prostate arteries using a 2.8 French microcatheter. The prostate was embolized with 300-500 $\mu \mathrm{m}$ Microspheres (Embosphere $\left.{ }^{\circledR}\right)$, using complete blood stasis as the end point. The prostate tissues were analyzed histologically to characterize the effects of the embolization.

Results: The embolic material within the prostate tissue was easily identified as homogeneous, bright eosin-red spheroids filling the vessel lumens. Ischemic necrosis surrounded or not by chronic inflammatory reactions containing macrophages were considered as a result of the artery embolization. Also, some aspects related to the healing process were observed being fibrotic nodules surrounded by glands with squamous metaplasia of the epithelial lining the most important. In the remaining sections, due to the precocious surgical intervention, the classic findings of BPH were still present with the glandular and stromal hyperplasia associated with nonspecific chronic prostatitis.

Conclusions: This is the first description of prostate histology in BPH patients treated by PAE, a new procedure that is being used increasingly as a therapeutic intervention. The recognition of the changes caused by this new modality of treatment has become a very important differential in a chronic granulomatous reaction of the prostate tissue.

\section{ARTICLE INFO}

\section{Key words:}

Prostatic Hyperplasia; Therapeutics; Arteries; Histology; Embolization, Therapeutic; Prostate

Int Braz J Urol. 2013; 39: 222-7

Submitted for publication: July 07, 2012

Accepted after revision: February 04, 2013

\section{INTRODUCTION}

Benign prostatic hyperplasia (BPH) is the most common benign neoplasm in men $(1,2)$. It typically occurs in the sixth and seventh decades, and more than $40 \%$ of men diagnosed with $\mathrm{BPH}$ present with the clinical manifestations (3-6). BPH causes obstructive urinary symptoms such as hesitancy, a decreased urinary stream, intermittency, sensation of incomplete emptying, nocturia, and 
an increase in frequency and urgency (3). Voiding difficulties attributable to BPH can be quantified with the International Prostatic Symptom Score. Various medications, specifically 5 - $\alpha$-reductase inhibitors and selective $\alpha$-blockers, can decrease the severity of voiding symptoms caused by BPH (3-6). Transurethral resection of the prostate (TURP) continues to be considered the best therapy for patients with BPH. Due to the relative morbidity of TURP, minimally invasive techniques have been developed as alternative treatments for BPH, including transurethral microwave thermotherapy and other laser-ablation therapies. However, open surgery, or TURP, represents the most common option for treating BPH (7-10). Taking into account the patients' comorbidities, surgical intervention in this group may be considered high-risk (6). Prostate artery embolization (PAE) has been used primarily to control massive hemorrhage after a prostatectomy or a prostate biopsy (11-15). PAE appears to be an acceptable and safe option to treat $\mathrm{BPH}$, and it is now emerging as an innovative treatment modality $(16,17)$. Here, we present the first description of prostate tissue histology in two patients who underwent TURP after PAE.

\section{MATERIALS AND METHODS}

Two male patients aged 67 and 68-years-old, with cardiovascular disease presented to the urology department with acute urinary retention due to $\mathrm{BPH}$. They underwent transurethral catheter drainage, and the digital rectal examinations revealed large prostates of greater than $50 \mathrm{~g}$ in both patients. The prostate specific antigen (PSA) levels in these patients were $7.1 \mathrm{ng} / \mathrm{ml}$ and $12.9 \mathrm{ng} / \mathrm{ml}$, respectively. Transrectal US showed an enlarged, heterogeneous prostate in each man. The prostates were $51 \mathrm{~g}$ and $63 \mathrm{~g}$ in volume, and the intravesical prostate protrusion measurements were 9 and $16 \mathrm{~mm}$, respectively. Both patients underwent prostate biopsies and each patient had 12 tissue cores removed, which upon examination exhibited the features of benign prostatic tissue.

In order to provide good orientation to the prostate site and related structures in the pelvis, we filled a Foley balloon in the bladder of each patient with contrast medium (a mixture of $10 \mathrm{~mL}$ of $50 \%$ iodinated contrast medium plus 50\% normal saline solution). Patients underwent angiography and embolization in the interventional radiology suite using the FD20 DSA unit (Philips, Netherlands) and with non-ionic Visipaque ${ }^{\circledR}$ contrast medium (Iodixanol $320 \mathrm{mg} \mathrm{I} / \mathrm{mL}$, GE, Healthcare, Europe). A $400 \mathrm{mg}$ intravenous dose of ciprofloxacin was given prior to the procedure followed by $500 \mathrm{mg}$ orally twice a day for seven days after PAE. Patients also received non-opioid analgesic and nonsteroidal anti-inflammatory medication after embolization, if necessary. Intervention was performed under local anesthesia through the right transfemoral approach. Initial pelvic angiography was performed to evaluate iliac vessels and the prostate arteries during arterial and late phases. Selective digital subtraction angiography of the right and left internal iliac arteries was performed with a 5-French Cobra 2 or Vertebral catheter to assess the blood supply to the prostate. Bilateral selective catheterization of the inferior vesical artery, superior vesical artery, obturator artery, middle rectal artery and internal pudendal artery was then performed using a microcatheter (Embocath ${ }^{\circledR}$; Biosphere Medical, USA or Progreat ${ }^{\circledR}$ 2.8, Terumo, Japan). Angiography was performed by manually injecting 3-5 mL of contrast medium to identify any blood supply to the prostate and to ensure that the tip of the microcatheter was inside or at the ostium of the prostatic arteries. When spasm occurred nitroglycerin was used. Tris-acryl microspheres (Embosphere ${ }^{\circledR}$ Microspheres, Biosphere Medical, Roissy, France) $300-500 \mu \mathrm{m}$ in diameter were used for embolization. We diluted the $2.0 \mathrm{~mL}$ Embosphere ${ }^{\circledR} \mathrm{Mi}$ crospheres syringe to a total volume of $22 \mathrm{~mL}$ using equal amounts of contrast medium and saline. The microsphere mixture was slowly injected under fluoroscopic guidance. Embolization of the prostatic arteries was performed to stasis, without reflux of the mixture to undesired arteries. Follow-up angiography was performed manually with the microcatheter using the power injector with the 5-F catheter at the anterior branch of the internal iliac artery to check for any further blood supply to the prostate. Embolization was then performed on the contralateral side by using the same technique.

One patient failed bilateral PAE twice. This patient was unable to void spontaneously when the indwelling urinary catheter was removed and the patient was referred to TURP after only 5\% reduction 
in the prostate size. The other patient was excluded from the PAE study protocol due to urodynamic findings and was submitted to TURP.

The two respective post-PAE specimens were fixed in formalin and submitted for histological evaluation according with the 2012 CAP protocol for the examination of TURP specimens $(18,19)$. All the tissue was submitted for evaluation in the first patient and 8 slides was reviewed. The tissue from the second patient was partially submitted and 10 slides were reviewed. The slides were stained with hematoxylin and eosin and were examined using an optical microscope by our pathology department staff on the first sign out. A clinical history of embolization was provided to the pathologist at the initial time of pathologic examination in both cases and the history of negative previous biopsies from both patients were also noticed at the time. Gross pathology reports were reviewed, and pertinent data were recorded. Routine sections from gross specimens were microscopically examined and reviewed by other 2 pathologists.

\section{RESULTS}

Surgical specimens were received in buffered formalin 10\%, consisting in prostate tissue measuring 6 and $8 \mathrm{~cm}$, weighting 14.5 and 36.0 grams for patients 1 and 2, respectively. The histological findings of the prostate samples were very similar for both patients and are illustrated in Figure-1. The embolic material was easily identified in both cases using standard hematoxylin and eosin staining as 2 to $3 \mathrm{~mm}$ homogeneous-appearing, brightly eosin-red spheroids. Despite the spheroids are the only specific finding on the specimens, we readily identified changes on the surrounding tissue due to the embolization, such as ischemic necrosis, that was surrounded or not by histiocytes, remembering a foreign body reaction rarely containing giant multinucleated cells. In the surrounding prostate tissue near the microspheres, there was identifiable vascular ectasia and moderate quantities of stromal lymphocytes. The transition between necrotic and normal prostate tissue was generally abrupt, and it was possible to detect a ribbon of neuthrophils, lymphocytes and proliferated fibroblasts delimitating the two areas. Also, a nodular fibrosis with hyalini- zation as a consequence of the healing process was present in some areas associated with squamous metaplasia of the epithelium lining the surrounding glands. All these alterations were found in about 5\% of the tissue examined in both cases. The remaining 95\% of the prostate tissue exhibited the classic findings of BPH, glandular and stromal hyperplasia, as well as mild, nonspecific chronic prostatitis. No high-grade intraepithelial neoplasia or adenocarcinoma was found in the slides of either patient.

\section{DISCUSSION}

Our report is the first to describe the histological features of the prostate after PAE. Although there are several reports that describe the post-treatment alterations in cancerous or non-cancerous prostate tissue following hormonal therapy, use of 5 - $\alpha$-reductase inhibitors or radiation (20), no post-PAE histological findings have been mentioned in the literature to date. With the continuing advances in the techniques and emerging therapies in $\mathrm{BPH}$ and cancer treatments, pathologists will play a central role in documenting the effects of these new treatment options on prostate tissue as well as a role in helping to improve these new methods. Although these findings are nonspecific and the only finding that confirms the attempted treatment BHP with PAE are the identification of the spheroids, the pathologist's role in this scenario is to inform the clinicians the histological finding of the spheroid, try to quantify the amount of necrosis to give a notion and feedback of the effectiveness of the modality of treatment and make differential diagnosis with other conditions that could present as an chronic granulomatous reaction, such as acute infarct of hyperplastic nodules, fibrosis in advanced stages of infarct in nodular hyperplasia surrounded by squamous metaplasia, granulomas following any kind of surgical intervention or instrumentation, granulomas secondary to the use of BCG in treatment of urothelial neoplasm, and idiopathic or infectious granulomatous prostatitis. All these diagnostic entities should be ruled out in a setting of chronic granulomatous inflammatory reaction. Certainly the clinical history and clinical information of each one of these conditions 
Figure 1 - Microscopic aspects of prostate tissue following PAE. (A) Embolic material used to occlude vessels represented by homogeneous, densely eosinophilic spheres. (B) Ischemic necrosis with no inflammatory reaction. (C) Fibrotic nodule surrounded by glands presenting squamous metaplasia of epithelial lining. (D-F) Extensive necrosis surrounded by histiocytes forming epithelioid granulomas.190x215 mm (96 x $96 \mathrm{DPI})$.
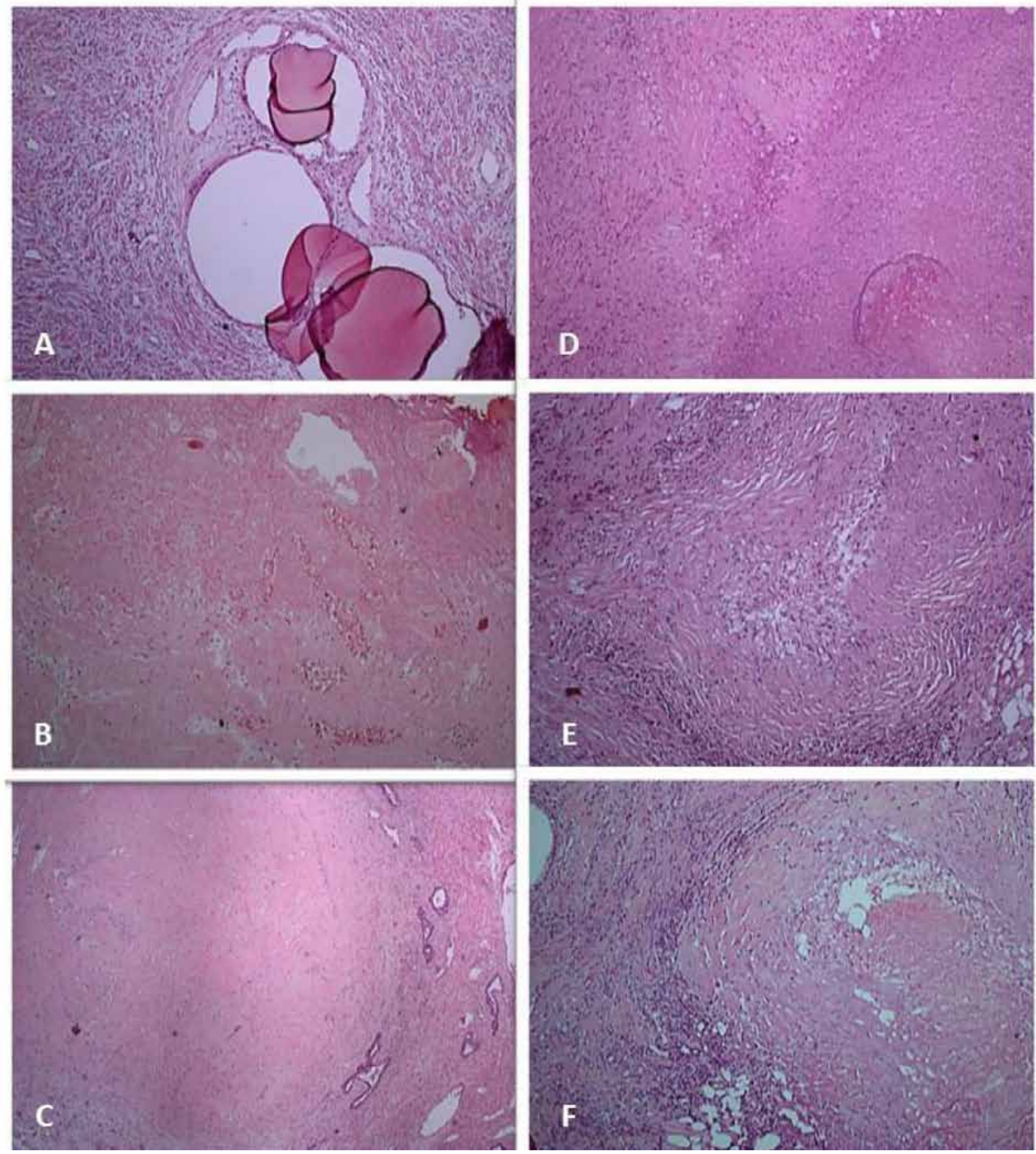

should be informed for the pathologist so that one can make a more accurate diagnostic. Arterial embolization has previously been used in cases of prostate hemorrhage following surgery or prostate biopsy (11-15), and recently PAE has been proposed as a new option to treat BPH $(16,17)$. Studies on pigs have reported decreases in prostate gland

size following PAE with no side effects, providing preliminary evidence that this approach is efficient and safe (21). We have previously described two cases of BPH successfully treated with PAE that resulted in a reduction in prostate size at 6 months of $47 \%$ in a patient undergoing a bilateral PAE and of $28 \%$ in a patient undergoing unilateral 
PAE (16). A continued reduction in prostate volume was observed during the 18 months follow-up with magnetic resonance imaging (MRI) showing a reduction of 54\% in the patient with the bilateral PAE. For the patient undergoing unilateral PAE, the reduction was stable at 6 months follow-up, and the patient experienced only mild urinary symptoms (22). Organ histology following embolization has been described in uterine leiomyomas. In these cases, the authors describe hyaline necrosis, dense fibrosis and a lack of inflammation (23). Further complications have been reported, the most dangerous including hematoma formation, fistulas, necrosis with secondary infection and septicemia and pseudoaneurysms responsible for significant hemorrhage. Although we do not know the long-term consequences of therapeutic artery embolization in the prostate, urologists and radiologists should remember the complications observed in the uteri as more patients undergo this new form of therapy $(24,25)$. Arterial embolization procedures have the potential to become increasingly common as a result of being minimally invasive. In order to avoid misdiagnoses, pathologists should be aware of the histological characteristics that distinguish post-PAE prostate tissue from $\mathrm{BPH}$, including extensive necrosis and granulomatous reaction. Further, the observation of these histological characteristics could prevent the possible complications that result from the vascular interruption in prostate tissue resulting in ischemia and necrosis.

\section{CONFLICT OF INTEREST}

None declared.

\section{REFERENCES}

1. Barry MJ: Measuring and assessing BPH in population groups. In Kirby RS, McConnell JD, Fitzpatrick Roehrborn JM, Roehrborn CG, Boyle P (ed.), Textbook of benign prostatic hyperplasia. Taylar \& Francis, London. 2005; pp. 141-50.

2. Issa MM, Fenter TC, Black L, Grogg AL, Kruep EJ: An assessment of the diagnosed prevalence of diseases in men 50 years of age or older. Am J Manag Care. 2006; 12: S83-9.
3. Emberton M, Andriole GL, de la Rosette J, Djavan B, Hoefner $\mathrm{K}$, Vela Navarrete R, et al.: Benign prostatic hyperplasia: a progressive disease of aging men. Urology. 2003; 61: 267-73.

4. Ziada A, Rosenblum M, Crawford ED: Benign prostatic hyperplasia: an overview. Urology. 1999; 3 (Suppl 3a): 1-6.

5. Guess HA, Arrighi HM, Metter EJ, Fozard JL: Cumulative prevalence of prostatism matches the autopsy prevalence of benign prostatic hyperplasia. Prostate. 1990; 17: 241-6.

6. AUA Practice Guidelines Committee: AUA guideline on management of benign prostatic hyperplasia (2003). Chapter 1 : Diagnosis and treatment recommendations. J Urol. 2003; 170: $530-47$

7. Roehrborn CG, Preminger G, Newhall P, Denstedt J, Razvi H, Chin LJ, et al:: Microwave thermotherapy for benign prostatic hyperplasia with the Dornier Urowave: results of a randomized, double-blind, multicenter, sham-controlled trial. Urology. 1998; 51: 19-28

8. Wheelahan J, Scott NA, Cartmill R, Marshall V, Morton RP, Nacey $\mathrm{J}$, et al.: Minimally invasive laser techniques for prostatectomy: a systematic review. The ASERNIP-S review group. Australian Safety and Efficacy Register of New Interventional Procedures--Surgical. BJU Int. 2000; 86: 805-15.

9. Roehrborn CG, Issa MM, Bruskewitz RC, Naslund MJ, Oesterling JE, Perez-Marrero R, et al:: Transurethral needle ablation for benign prostatic hyperplasia: 12-month results of a prospective, multicenter U.S. study. Urology. 1998; 51: 415-21. Erratum in: Urology. 1998; 52: 159.

10. Cioanta I, Muschter R: Water-induced thermotherapy for benign prostatic hyperplasia. Tech Urol. 2000; 6: 294-9.

11. Mitchell ME, Waltman AC, Athanasoulis CA, Kerr WS Jr, Dretler SP: Control of massive prostatic bleeding with angiographic techniques. J Urol. 1976; 115: 692-5.

12. Appleton DS, Sibley GN, Doyle PT: Internal iliac artery embolisation for the control of severe bladder and prostate haemorrhage. Br J Urol. 1988; 61: 45-7.

13. Michel F, Dubruille T, Cercueil JP, Paparel P, Cognet F, Krause $D$ : Arterial embolization for massive hematuria following transurethral prostatectomy. J Urol. 2002; 168: 2550-1.

14. Barbieri A, Simonazzi M, Marcato C, Larini P, Barbagallo M, Frattini $A$, et al.: Massive hematuria after transurethral resection of the prostate: management by intra-arterial embolization. Urol Int. 2002; 69: 318-20.

15. Rastinehad AR, Caplin DM, Ost MC, VanderBrink BA, Lobko I, Badlani GH, et al.: Selective arterial prostatic embolization (SAPE) for refractory hematuria of prostatic origin. Urology. 2008; 71: 181-4.

16. Carnevale FC, Antunes AA, da Motta Leal Filho JM, de Oliveira Cerri LM, Baroni RH, Marcelino AS, et al.: Prostatic artery embolization as a primary treatment for benign prostatic hyperplasia: preliminary results in two patients. Cardiovasc Intervent Radiol. 2010; 33: 355-61. 
17. Carnevale FC, da Motta-Leal-Filho JM, Antunes AA, Baroni RH, Freire GC, Cerri LM, et al.: Midterm follow-up after prostate embolization in two patients with benign prostatic hyperplasia. Cardiovasc Intervent Radiol. 2011; 34: 1330-3.

18. Humphrey PA, Walther PJ: Adenocarcinoma of the prostate. I. Tissue sampling considerations. Am J Clin Pathol. 1993; 99: 746-59.

19. Merrimen JL, Jones G, Walker D, Leung CS, Kapusta LR, Srigley JR: Multifocal high grade prostatic intraepithelial neoplasia is a significant risk factor for prostatic adenocarcinoma. J Urol. 2009; 182: 485-90; discussion 490.

20. Evans AJ, Ryan P, Van derkwast T: Treatment effects in the prostate including those associated with traditional and emerging therapies. Adv Anat Pathol. 2011; 18: 281-93.

21. Sun F, Sánchez FM, Crisóstomo V, Lima JR, Luis L, GarcíaMartínez V, et al.: Benign prostatic hyperplasia: transcatheter arterial embolization as potential treatment--preliminary study in pigs. Radiology. 2008; 246: 783-9.

22. Carnevale FC, da Motta-Leal-Filho JM, Antunes AA, Baroni RH, Freire GC, Cerri LM, et al.: Midterm follow-up after prostate embolization in two patients with benign prostatic hyperplasia. Cardiovasc Intervent Radiol. 2011; 34: 1330-3.
23. Kuzel D, Mara M, Horak P, Kubinova K, Maskova J, Dundr $P$, et al.: Comparative outcomes of hysteroscopic examinations performed after uterine artery embolization or laparoscopic uterine artery occlusion to treat leiomyomas. Fertil Steril. 2011; 95: 2143-5.

24. Takeda A, Kato K, Mori M, Sakai K, Mitsui T, Nakamura $\mathrm{H}$ : Late massive uterine hemorrhage caused by ruptured uterine artery pseudoaneurysm after laparoscopic-assisted myomectomy. J Minim Invasive Gynecol. 2008; 15: 212-6.

25. Aungst M, Wilson M, Vournas K, McCarthy S: Necrotic leiomyoma and gram-negative sepsis eight weeks after uterine artery embolization. Obstet Gynecol. 2004; 104: 1161-4.

Correspondence address:

Dr. Katia Ramos Moreira Leite

Department of Radiology

Laboratory of Medical Research, LIM55

University of Sao Paulo Medical School

Av. Dr. Arnaldo 455 / 2145

Sao Paulo, SP, 01246-903, Brazil

Telephone: +55 11 3061-7183

E-mail: katiaramos@uol.com.br 